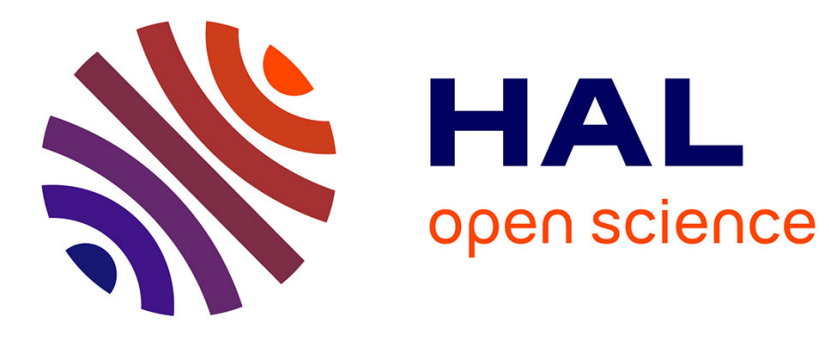

\title{
Construire des savoir-faire perceptifs en apnée Mary Schirrer
}

\section{To cite this version:}

Mary Schirrer. Construire des savoir-faire perceptifs en apnée: Une méthode de réflexivité en action coach-pratiquant. Movement \& Sport Sciences - Science \& Motricité, 2018, 1 (99), pp.35-46. 10.3917/sm.099.0035. hal-02946113

\section{HAL Id: hal-02946113 \\ https://hal.univ-lorraine.fr/hal-02946113}

Submitted on 22 Sep 2020

HAL is a multi-disciplinary open access archive for the deposit and dissemination of scientific research documents, whether they are published or not. The documents may come from teaching and research institutions in France or abroad, or from public or private research centers.
L'archive ouverte pluridisciplinaire HAL, est destinée au dépôt et à la diffusion de documents scientifiques de niveau recherche, publiés ou non, émanant des établissements d'enseignement et de recherche français ou étrangers, des laboratoires publics ou privés. 
Construire des savoir-faire perceptifs en apnée

Une méthode de réflexivité en action coach-pratiquant

Mary SCHIRRER

LISEC EA 2310, Faculté des Sciences du Sport de Nancy

Schirrer, M. (2018). Construire des savoir-faire perceptifs en apnée-Une méthode de réflexivité en action coach-pratiquant. Movement \& Sport Sciences-Science \& Motricité, 99, $35-46$.

\section{Résumé :}

Progresser en apnée suppose notamment d'apprendre à écouter les signes subtils ou violents envoyés par le corps. Cette recherche analyse les difficultés et stratégies des pratiquants et entraîneurs pour s'approprier ce corps vivant qui réagit et s'adapte à l'environnement aquatique et au stress généré par la situation d'apnée. Le cadre théorique est celui de la phénoménologie et de l'anthropologie sensorielle. La démarche est ethnographique.

L'enjeu de l'article est double. D'une part rendre compte de l'activité d'apnéistes ordinaires à partir d'observations participantes. Il s'agit d'identifier comment ils apprennent à ressentir et à se repérer parmi cette diversité de sensations corporelles qui émergent de la situation d'apnée ; quelles techniques et quelles procédures favorisent la construction de savoir-faire perceptifs relatifs aux sensations corporelles. D'autre part présenter et analyser une méthode de réflexivité en action initiée par un entraîneur afin de co-construire, avec l'athlète, une échelle esthésiologique, en caractérisant les indices significatifs pour le sujet engagé dans son apnée, ainsi qu'une stratégie permettant de dépasser des blocages éventuels.

Finalement, ce travail invite à révéler les savoir-faire et l'expertise d'acteurs engagés sur le terrain afin d'améliorer certains processus perceptifs, au service de performances améliorées et/ou d'une meilleure gestion de son capital corporel.

Mots clés : corps vécu ; ressentis ; éducation sensorielle ; compétence perceptive ; apnée.

Je remercie vivement l'entraîneur Didier Zaenger qui m'a fait partager la vie de son club ainsi que sa démarche d'exploration de l'apnée. 


\title{
Building perceptual know-hows in freediving A method of reflexivity in action between the coach and the practitioners
}

\begin{abstract}
:
Making progress in freediving supposes in particular learning how to listen to the subtle or violent signs sent by the body. This research analyzes the difficulties and the strategies implemented by freedivers and coaches to appropriate this living body which reacts and adapts itself to the aquatic environment and to the stress generated by the situation of apnea. The conceptual framework is the one of phenomenology and sensory anthropology. The approach is an ethnographical one. The purpose of the article is double. On the one hand, reporting the activity of ordinary freedivers, from participating observations. The purpose is to identify how they learn to feel and to find their way among this diversity of physical sensations which emerge from the situation of apnea; Which techniques and which procedures favor the construction of perceptive know-hows connected with physical sensations. On the other hand, presenting and analyzing a method of reflexivity in action introduced by the coach, in order to co-build, with the athlete, a sensitive scale, by characterizing the significant indications felt by the subject committed in his apnea, as well as a strategy allowing him to overtake possible hang-ups. Finally, this work is an invitation to reveal the know-hows and expertise of ground-actors committed to improve some perceptive processes, in the service of improved performances and/or of a better management of his physical capital.
\end{abstract}

Keywords: lived body; feelings; sensory education; perceptive competence; freediving. I deeply thank coach Didier Zaenger who shared with me the life of his club as well as his approach of freediving. 
L'apnée attire des contemporains de plus en plus nombreux (Fricker, 2015), en quête de détente et de «reconnexion avec soi-même » (Schirrer, 2015a). En effet, l'activité attire pour le bien être qu'elle procure, la détente, l'apprentissage de la respiration, l'exploration de soi et du milieu aquatique qu'elle autorise. L'apnée est une pratique qui élargit ou plutôt redessine la « carte des sens » des individus, par la prédominance de la proprioception et l'émersion de sensations nouvelles (Schirrer, 2015b, 2015c).

L'engouement pour l'apnée n'est pas sans rapport avec l'engouement contemporain pour les pratiques physiques et récréatives de pleine nature, immersives, permettant de fuir, pendant une durée plus ou longue, un monde civilisé, "pasteurisé » et pollué (Andrieu, 2014a ; Corneloup, 2011 ; Kalaora, 2001). Dans les stages de survie en pleine nature, Pasche vise "une immersion primitive et une autonomie sauvage» (Pasche, Bertrand, 2013) où les individus (ré)apprennent à écouter et à sentir la nature pour mieux s'y adapter. Dans son analyse de l'Ultra-Trail-Mont-Blanc (UTMB), Bessy (2012) a pu montrer combien l'intensité et la durée du parcours (perte des repères temporels, fatigue, stress, souffrance) extrémisent les limites connues du corps, générant un nouveau rapport sensible à la nature. Chavaroche (2018) explore la sensorialité développée par les pratiquants de slackline pour maintenir un équilibre éphémère. Dans une analyse socio-anthropologique du surf, Sayeux (2008) explore la sensorialité des surfeurs dans cette immersion écologique (Andrieu, 2014a) où tous les sens sont mobilisés. Le "savoir-faire » en tant que disposition sensible du surfeur, s'appuie en premier lieu sur la vision. Un long apprentissage du regard permet de lire les vagues. Mais lorsque l'œil ne suffit pas, lorsque le surfeur est sous l'eau (chute ou canard), l'aspiration et le refoulement des vagues, la mousse ou l'eau compacte, deviennent autant de repères tactiles pour s'orienter et décider de rester sous l'eau ou de mettre fin à l'apnée. L'auteure explore alors ces «savoir-faire sensibles » qui nécessitent un long temps d'apprentissage afin d'affiner les ressentis et améliorer la gestion de la pratique.

Les apnéistes experts évoquent un voyage intérieur au plus près du corps ${ }^{1}$, pour rentrer au plus profond de leurs sensations et découvrir comment leur corps fonctionne et s'adapte ${ }^{2}$. La situation d'apnée conduit à des adaptations du corps vivant (Andrieu, 2016) en interaction dynamique avec l'environnement aquatique : l'écologie motrice (Andrieu, 2013a). Certaines de ces adaptations sont rapidement perceptibles (diaphragme ou glotte qui bougent). Des sensations nouvelles émergent, quand d'autres adaptations sont très subtiles, voire obscures à la conscience (circulation sanguine qui se concentre sur les « organes nobles »).

Progresser en apnée suppose d'améliorer sa capacité à retenir son souffle, à se déplacer de façon économique dans une position hydrodynamique, à gérer son stress (Lemaître, 2011), à apprendre à se situer dans sa performance, notamment en écoutant les signes subtils ou violents envoyés par le corps, pour mieux les interpréter, les accepter ou les dépasser, afin de poursuivre sa performance ou au contraire y mettre fin et éviter de se mettre en danger (éviter la syncope anoxique).

A partir d'une démarche inductive et qualitative seront analysées les difficultés et stratégies des pratiquants et entraîneurs pour s'approprier ce corps vivant qui réagit et s'adapte à l'environnement et au stress généré par la situation d'apnée. A partir d'observations participantes, il s'agit d'identifier comment les pratiquants, notamment débutants, apprennent à ressentir et à se repérer parmi cette diversité de sensations corporelles qui émergent de la situation d'apnée. Enfin, une démarche de terrain, ici une méthode de réflexivité coach-

\footnotetext{
${ }^{1}$ Interview de Loïc Leferme pour le journal L'Équipe, $\mathrm{n}^{\circ}$ 1210, août 2005.

${ }^{2}$ Pierre Frolla interviewé par Gilles Raveneau (2006).
} 
pratiquant inspirée de la Programmation Neuro Linguistique (PNL), est présentée. Cette méthode originale permet au coach de recueillir l'expérience subjective de l'apnée chez différents pratiquants, pour ensuite identifier avec eux leur cheminement mental et leur expérience corporelle. Ensemble, ils co-construisent une échelle esthésiologique ${ }^{3}$, ainsi qu'une stratégie permettant de dépasser des blocages éventuels, tester des inducteurs ou images.

\section{Cadre théorique}

On considère communément que deux sources d'informations permettent à l'homme de s'orienter dans le monde :

-celles qui lui parviennent de l'environnement où il évolue par ses sens extéroceptifs (les cinq sens classiquement reconnus : vision, audition, olfaction, goût et toucher) ;

- celles qui lui parviennent de l'intérieur de lui-même par des récepteurs sensoriels situés dans les différents tissus et tout un ensemble de cellules constituant la proprioception (capteurs musculaires et articulaires, capteurs de force, capteurs vestibulaires) et l'intéroception (informations afférentes des viscères ou du système respiratoire). Le tout renvoyant à la somesthésie, ou sensibilité du corps, c'est-à-dire la perception sensorielle qui passe par le corps lui-même et non par les organes des sens « individuels ».

La sensation résulte de la stimulation, externe ou interne, des récepteurs sensoriels. Elle peut donc rester à l'état diffus. La perception renvoie à ce qui est perçu de la sensation. Elle résulte de l'interprétation et du jugement que nous faisons à partir de la sensation. Elle est un savoir connaissant et sensible. Le terme ressenti renvoie plus communément à la capacité d'une personne à se rendre attentive à ses sensations internes. Si les perceptions sont orientées par les potentialités des récepteurs, les significations diverses acquises par chaque individu au cours de son existence interviennent aussi (Schirrer \& Paintendre, 2017). Qualité intrinsèque de tout tissu vivant, la sensibilité est aussi cette capacité ou disposition de l'homme à être affecté par ses sens, par des variations de ses états internes ou de certains aspects de l'environnement (Gaillard, 2011 ; Récopé, 2001).

Dans une approche holistique du corps, Andrieu distingue le corps vivant, le corps vécu et le corps décrit (Andrieu, 2013a, 2013b). Par le concept de corps vivant, il pointe le corps en acte avant que la conscience s'en aperçoive (Berthoz \& Andrieu, 2011). Ce corps vivant, et mouvant, est constamment en interaction dynamique avec son environnement. L'écologie motrice renvoie à cette interaction sensible et spontanée du corps avec ses environnements, ici le milieu aquatique. Une petite partie seulement de notre activité corporelle parvient à notre conscience. De nombreux mouvements apparaissent en dessous de la conscience et ce qui se passe à l'intérieur de notre corps (modifications chimiques ou physiologiques par exemple) est très rarement perceptible ou conscientisable (mouvement de la glotte, taux de $\mathrm{CO} 2$ qui augmente par exemple). Néanmoins, un ensemble de sensations émanent des organes du corps vivant par le biais de la perception. Pour Andrieu et Burel (2014), «il [le corps vécu] opère un tri entre les différentes activations somatiques issues du corps vivant selon qu'elles appellent ou non une attention particulière. ». Ce corps vécu est la traduction de la sensibilité du vivant après perception, soit conscientisation et donc interprétation subjective des informations corporelles internes. A un dernier seuil, l'état de conscience (Andrieu, 2016) est plein, entier. Le corps décrit représente la possibilité du sujet à traduire, c'est-à-dire mettre en

\footnotetext{
${ }^{3}$ Entendue comme une échelle de ressentis personnelle et nuancée, caractérisant les indices significatifs pour le sujet engagé dans son apnée.
} 
mots (avec toutes les difficultés relatives notamment à l'accessibilité du vocabulaire du corps), son état sensationnel, interne voire émotionnel.

En situation d'apnée, le corps est en interaction sensible et spontanée avec le milieu aquatique. Il s'adapte à la situation d'apnée (hypercapnie et hypoxie notamment) et au milieu aquatique (effets de la pression hydrostatique, de la poussée d'Archimède, bradycardie, etc.). Le défi de l'apnéiste consiste à s'ouvrir le plus possible à certaines adaptations ou signaux envoyés par son «corps vivant » notamment en développant une écoute attentive dirigée vers son intériorité ; apprendre à mieux se connaître en construisant des repères sur soi permettant de sortir lucide et éviter une syncope.

L'anthropologie et la sociologie éclairent également l'activité perceptive de l'homme. Anthropologues et sociologues s'intéressent aux usages des sens au sein d'une culture ou d'un milieu social. «La configuration des sens, la tonalité et le contour de leur déploiement, sont de nature sociale et non seulement physiologique »(Le Breton, 1992, p. 67). La vue et les couleurs font par exemple l'objet d'une socialisation plus poussée, au contraire de l'expérience olfactive (Candau, 2005). Vigarello (2014), à travers une histoire de la perception, relève que les individus travaillent actuellement sur la perception de leur corps, avec pour objectif de la transformer pour se transformer eux-mêmes. Nous serions passés d'un «je pense donc je suis », à un «je sens donc je suis».

Envisagée en tant que processus, la sensibilité pourrait se modeler, s'actualiser, s'ajuster sans cesse (Wathelet, 2013). Différentes études s'intéressent à la perception comme acte, afin de mettre en avant des processus de "socialisation de l'attention », d'éducation de la sensibilité. Becker (2012) souligne le rôle des pairs dans la socialisation de l'attention et l'élaboration d'un jugement perceptif chez les fumeurs de marijuana. L'impact du langage dans les processus perceptifs fait quant à lui débat. L'anthropologie sensorielle apporte les preuves d'une «indépendance de la sensation sur le langage : une personne sait reconnaitre une odeur, un goût, etc. et lui attribuer une valeur affective, sans pour autant savoir les nommer » (Gélard, 2013, p. 15). Pourtant, les mots organisent aussi les catégories qui nous servent à connaître le monde et à partager cette connaissance. L'hypothèse d'une influence linguistique « relative » est globalement acceptée. C'est-à-dire que la dénomination participe en partie de la perception. À propos de la perception olfactive, Candau (2005) note que les êtres humains sont plutôt habiles pour détecter les odeurs, modestement compétents pour les discriminer, beaucoup moins performants pour les décrire. Mais le partage et les échanges avec autrui facilitent cette identification: "Les sensations se structurent alors d'autant plus facilement sur un fond de paysage commun que se produit un effet de conformité avec le groupe, véhiculé évidemment par le langage» (Candau, 2005). Les métaphores et analogies permettent de nommer et partager l'indicible. Enfin, la non disponibilité immédiate des mots rendrait indispensable le recours à l'autre pour que l'altérité co-construise la singularité du sens. Wacquant (1989) souligne l'importance de la médiation langagière sur le ring des boxeurs : apprentissage des noms techniques, corrections, encouragements, commentaires, conseils, comparaisons et métaphores, autant de formes discursives subtiles donnant à voir à ou à sentir tel ou tel mouvement ou posture.

Finalement, ces travaux permettent d'envisager comment l'individu apprend de nouvelles configurations sensorielles, de nouveaux usages et langages des sens ; comment il construit un « savoir-faire perceptif » (olfactif, auditif ou sensible) c'est-à-dire cette capacité à utiliser sa sensorialité pour agir et prendre des décisions (Schirrer \& Paintendre, 2017). Les individus vivent au quotidien des modelages sensoriels, explicites ou implicites. Ils développent des «géographies de la sensibilité » (Wathelet, 2013) souvent adaptées à une pratique 
particulière. Comment ces apprentissages sensoriels sont-ils mis en œuvre sur le terrain de l'apnée ? Comment les entraîneurs ${ }^{4}$ facilitent-ils le repérage sensoriel de leurs pratiquants ?

L'enjeu de ce travail consiste à identifier et comprendre les processus d'éducation et d'apprentissage de la sensibilité ; identifier quelles sont les pratiques, difficultés, stratégies mises en œuvre de façon plus ou moins conscientes afin de mieux percevoir/écouter son corps.

\section{Méthodes}

La démarche de recherche est avant tout ethnographique. Nous avons ainsi pratiqué pendant trois années dans un club d'apnée de l'Est de la France ${ }^{5}$, au sein du groupe compétition (niveau départemental à national). Durant ces trois années (2013 à 2016), un journal de bord a été tenu, où étaient consignés les entraînements, réactions, interactions entre apnéistes. Nous avons consigné nos propres apprentissages, interactions entre pairs et avec l'entraîneur. Le groupe «débutants » a été observé pendant cette même période. Nous avons assisté aux cours théoriques et tenu également un journal de bord. Enfin, nous avons participé à 4 compétitions de niveau départemental à régional, et suivi ainsi les compétiteurs du club. Les activités observées avaient lieu en piscine exclusivement (apnée statique et dynamique). L'enjeu de ces observations participantes était bien de mettre en œuvre une forme d'ethnopraxie au sens de Wacquant, forme d'observation qui consiste à "pratiquer en temps et en situation réels avec les indigènes de sorte à acquérir, comme eux, par la routine, les savoirs tacites et les catégories de perception qui composent leur univers» (Wacquant, 1989: 80). Afin de compléter le journal de bord et ces observations participantes, neuf entretiens ont été réalisés : 2 entretiens semi-directifs avec les entraîneurs, Didier pour le groupe "compétition », Hervé pour le groupe «initiation»; 7 entretiens «mixtes » avec des pratiquants issus des deux groupes, volontaires et tous engagés dans une recherche de progrès, ayant pour cinq d'entre eux participé à au moins une compétition. La syncope n'était pas retenue ici comme critère de sélection, mais il s'est avéré que six des sept interviewés en avaient déjà vécu au moins une, en compétition ou à l'entraînement. La syncope est le signe du dépassement de ses propres limites, une reprise de contrôle du corps vivant qui « déconnecte » pour mieux se protéger en situation d'anoxie (Schirrer, 2018). Les entretiens mixtes comprenaient deux parties. Dans un premier temps, l'entretien d'explicitation (Petitmengin, 2004 ; Vermersch, 1993) devait amener l'interviewé à explorer rétrospectivement son expérience (une apnée choisie parce qu'elle était très agréable, ou extrêmement difficile ; une syncope). Il s'agissait de documenter le plus précisément possible le vécu du sujet, accéder à des informations "pré-réfléchies » conscientisables. Pour cela, et dans la mesure du possible, l'entretien était réalisé dans l'heure qui suivait la contre-performance (deux pratiquants). Le questionnement n'était que descriptif, orienté vers la structure du vécu («Par quoi avez-vous commencé ?» «Et ensuite qu'avezvous fait? ») ou le contenu du vécu («Quand vous nagiez, que faisiez-vous ? » «À quoi étiezvous attentif alors ? »). Nous souhaitions ainsi documenter finement le processus d'écoute de

\footnotetext{
${ }^{4}$ Nous considérerons ici les termes « entraineur » et « coach » comme interchangeables, afin d'alléger l'écriture. Mais nous sommes conscients des nuances que nous occultons.

${ }^{5}$ Ancienne pratiquante (2000 à 2002), j'ai, dix années plus tard, suivi le chemin " classique » d'un " apnéiste ordinaire ", attentive à toutes les étapes qui scandent l'apprentissage de ce savoir-faire aquatique. J'ai suivi et validé les premiers enseignements théoriques, participé aux cours d'initiation, pour accéder ensuite au groupe compétition. Pour faire partie de ce groupe, les apnéistes doivent s'engager à participer à au moins une compétition au cours de l'année. J'ai donc participé à quatre compétitions dans l'Est de la France. Dans ce groupe, les pratiquants ont des niveaux hétérogènes, de départemental (qui commencent en compétition) à international avec Fa (55 ans, vice-championne du monde).
} 
soi, les sensations éventuellement ressenties, le cheminement mental. Une seconde partie était semi-directive. Il s'agissait d'explorer la pratique de l'année, les plaisirs et déplaisirs, intérêts et motivations, le parcours sportif de l'apnéiste, le rapport au corps et au dépassement de soi. Enfin, plusieurs entretiens ont été approfondis avec un des entraîneurs (Didier) à propos de la méthodologie qu'il a créée afin de faciliter ce repérage corporel des pratiquants.

Mobilisant une stratégie narrative, une première partie de résultats présentera les conduites typiques de débutants en apnée pour analyser les premiers apprentissages sensoriels mis en œuvre. Une seconde partie de résultats analysera la démarche de ce professionnel de terrain afin d'améliorer le repérage sensoriel du pratiquant.

\section{Débuter en apnée : déprivation sensorielle et premiers savoir-faire perceptifs}

En apnée statique, le pratiquant ne bouge pas. Il ne cherche pas à parcourir une longue distance sous l'eau. La technique de déplacement n'est donc pas un facteur de performance. L'apnéiste doit «attendre » le plus longtemps possible. L'augmentation du taux de CO2 dans son corps, la diminution du taux d'O2, l'absence de mouvements thoraciques, l'absence de circulation d'air dans la trachée, amènent peu à peu le «corps vivant » à se manifester : envie de respirer voire « soif d'air », spasmes au niveau du diaphragme, mouvements de la glotte. Si l'apnéiste poursuit (en ignorant ou acceptant ces différents signaux) d'autres sensations, plus « extrêmes » se manifesteront peu à peu (ces manifestations sont variables en fonction des sujets, plus ou moins diffuses): picotements dans les extrémités, sensation de bien-être inhabituel, lourdeur extrême dans les muscles (peu le cas en statique), vertiges, troubles visuels, tremblements.

En apnée dynamique, le pratiquant doit parcourir la plus longue distance en restant à environ un mètre sous la surface. Les débutants utilisent le plus souvent des bi-palmes et s'initient ensuite à la monopalme. Au cours d'une apnée dynamique, le pratiquant doit veiller à entretenir une propulsion constante, maintenir une position hydrodynamique, rester relâché, se repérer visuellement afin d'amorcer les virages, lâcher prise par rapport à certaines sensations («soif d'air », douleurs dans les jambes) tout en maintenant une certaine vigilance afin d'identifier quand mettre fin à son apnée. Aux sensations évoquées précédemment s'ajoutent les douleurs dans les jambes, liées à la dépense énergétique en situation d'hypoxie.

Les débutants observés en apnée nagent sous l'eau et sortent aux premiers signes d'inconfort. Ils focalisent leur attention sur les premières sensations liées à leur interaction avec le milieu aquatique : l'eau dans les oreilles, l'eau susceptible de rentrer dans le masque (alors on le serre le plus possible), les crampes qui apparaissent dans les jambes suite à la nage avec palme. En revanche, les débutants ne semblent pas discriminer différentes sensations corporelles. Ils sortent parce que «ils ne pouvaient plus tenir » (Hervé).

Le travail d'Hervé, l'entraineur dans ce club, est dans un premier temps le plus individualisé possible. Il questionne systématiquement: "Qu'est-ce qui t'a fait sortir? Qu'as-tu ressenti ? » Il cherche à placer les pratiquants dans une dynamique de construction de repères et d'exploration de soi. Il apporte peu à peu des connaissances. Tout d'abord l'existence de deux phases en apnée : phase d'aisance et phase d'inconfort. Les progrès en apnée semblent passer par cet aller-retour constant entre l'expérience et les explications/interprétations apportées par l'entraîneur ou les pairs, qui invitent progressivement à explorer cette zone d'inconfort, pour y construire de nouveaux repères et des significations : "ça me met des mots », " je comprends mieux les différents stades » (Da, 48 ans, débutante).

La médiation langagière apparaît essentielle afin d'aider les pratiquants à analyser ce qu'ils ont vécu et dépasser le «je n'en pouvais plus», «c'était trop dur». Ainsi, aux premiers 
virages à $25 \mathrm{~m}$, les débutants ont le sentiment d'avoir « dépassé le mur du son », avoir piloté ou dépassé leur corps. Pour Hervé, ils « sortent de la nage sous l'eau pour entrer réellement dans l'apnée », avec son lot de stress lié à la «soif d'air », de sensations corporelles liées à l'augmentation du taux de $\mathrm{CO} 2$ (hypercapnie) et la diminution du taux d'O2 (hypoxie).

Da revient sur sa première compétition en apnée dynamique, révélant la complexité du langage interne mêlé à l'identification de sensations contradictoires qu'elle commence à discriminer : spasmes, angoisse, être bien, être abandonnée.

$\mathrm{Da}$ : « au début, je commence à entrer dans le bassin, à 30 secondes en général je pars, mais là je n'arrive pas à respirer, c'est sec dans ma gorge, j'enlève le pince nez, je reprends ma respiration, j'avale. On entre dans le $20 \mathrm{~s}$, dans le $10 \mathrm{~s}$. Et là c'est la première fois ! Je suis poussée... et j'arrive au 25, je ne suis pas bien, j'ai des spasmes, et je me dis : «tu as fait une belle performance, vas jusqu'au mur au moins, allez ! Fais le pour ton fils ! »

Chercheur: des spasmes?

$\mathrm{Da}$ : je sentais une pression, qu'on a quand on a fini déjà $50[\ldots]$ je vois le carrelage, je vois le blanc qui arrive. Et ça y'est j'ai fait 25 , et je me dis : «allez c'est ridicule 25 , vas-y, ton fils va être content de toi, et j'arrive au mur et je suis bien ! »

$\mathrm{Ch}$ : et donc de 25 à 50 ?

Da : c'est passé ! à $10 \mathrm{~cm}$ je vois le mur, et là je me dis : «ah c'est bien finalement, j'ai encore de la marge, c'était juste un spasme, c'est l'angoisse ». Et là je glisse. En fait pour moi, c'était une belle performance, jusqu'au moment où j'ai perdu la notion de mur, la notion de taille de bassin, j'ai perdu cette notion-là, de repères... y'en a qui regardent, et moi j'étais complètement abandonnée ».

Au fil des séances, des exercices particuliers permettent d'accentuer certaines sensations, facilitant ainsi cette écoute du corps en action. Ainsi, réaliser une apnée statique de dix à vingt secondes puis enchaîner, sans reprise d'air, avec une apnée dynamique de $25 \mathrm{~m}$. L'entraîneur cherche ici à calmer les pratiquants afin qu'ils réalisent l'apnée dynamique le plus sereinement possible. En effet, l'apnée statique et l'immersion associée contribuent à abaisser le rythme cardiaque, ce qui profite à l'apnée dynamique qui suit. L'enjeu pour Hervé consiste à amener le pratiquant à s'y rendre attentif, à identifier peu à peu cet état d'apaisement et ce rythme cardiaque ralenti (qui sont finalement des facteurs de performance) pour conserver cet état pendant leur apnée dynamique.

Inversement, enchainer des apnées dynamiques de $25 \mathrm{~m}$, avec très peu de récupération entre chaque apnée (de quelques secondes à un seul cycle ventilatoire), amplifie l'impression de soif d'air et certaines sensations corporelles. Les jambes brûlent, elles sont dures. Ces situations contrastées, au cours desquelles les sensations sont «extrémisées », facilitent dans un premier temps leur perception par les pratiquants. Ensuite, avec les pairs et l'entraîneur, le pratiquant peut alors leur donner du sens. Enfin, comme c'est le cas avec l'hypercapnie, il s'agit aussi de s'habituer à ce type de sensations, les identifier comme un moment normal au cours de la réalisation de la performance, les accepter et lâcher prise.

Finalement, le pratiquant construit sa propre échelle esthésiologique: lors d'apnées rapprochées, quelles sont les sensations ressenties ? Lors d'une apnée dynamique après une courte apnée statique, quel état corporel puis-je identifier ? Il apprend à mettre en relation ses propres sensations, de plus en plus nuancées, avec des effets ou dimensions de sa performance, avec des paramètres de son activité (avoir réalisé son apnée avec peu ou beaucoup de récupération, poumons vides ou pleins, etc.).

Le partage des ressentis, les discussions avec les partenaires d'entraînement ou le coach, à l'issue d'une apnée longue, permettent d'apprendre à identifier ces signaux, à mettre en mots 
les sensations : «comment tu étais ? », «pourquoi es-tu sorti ? », « qu'est-ce qui t'a fait sortir ? ». Sachant qu'il est bien souvent très difficile pour l'apnéiste de savoir ce qui l'a fait sortir lorsqu'il débute ou lorsqu'il se situe dans une performance proche de sa limite. "Je ne pouvais plus tenir » (So, 35 ans, débutante). Er (45 ans, compétiteur de niveau départemental) nous confiait : "c'est mon corps qui est sorti», He (45 ans, compétiteur de niveau départemental) revient sur sa syncope : "C'est vraiment l'impression que tu nages, tu as forcé, et crac! Ça pète, ça se détraque, complètement. Je nage, je nage, de moins en moins bien... et d'un coup, ça casse d'un coup ! [...] C'est vraiment, tout s'arrête et il faut agripper quoi!»

Aux plus aguerris, l'entraîneur présente non plus deux, mais quatre phases d'une apnée, nouvelle grille de lecture de son expérience corporelle et sensible :

1. Une phase de confort : l'apnéiste essaie de cultiver un état de détente en limitant l'activité mentale. Avec l'entraînement, il cherche à la prolonger le plus possible.

2. Une phase de travail: les premières sensations d'inconfort apparaissent; le raisonnement conscient permet à l'apnéiste de contrôler et d'accepter ces sensations.

3. Une phase d'effort: les signes d'inconfort deviennent plus prégnants voire douloureux. Les apnéistes ressentent ici cette intense «soif d'air»si caractéristique : « Ça pique », «Ça chauffe », « Ça tire » (propos communément entendus et partagés en situation).

4. Une phase de lutte : les apnéistes s'engagent parfois en compétition. L'organisme envoie des signaux forts, douloureux, voire violents. L'apnéiste peut être tenté de dépasser ses limites. L'envie de respirer est au plus haut. C'est une zone critique imposant une vigilance extrême. Certains apnéistes y témoignent d'une nouvelle aisance «Ça redevient plus facile et ça passe super vite!». Cette phase est donc risquée car avec l'hypoxie sévère, la lucidité diminue et la «bascule » vers la perte de conscience est imminente.

Cette modélisation partagée permet aux pratiquants d'explorer et structurer leurs sensations, sur un «fond de paysage commun » (Candau, 2005). Ce «cadrage sensoriel » ainsi construit peut contribuer à une convergence relative des perceptions, mais surtout à une focalisation et une interprétation progressivement partagée. Bien entendu, ces quatre zones ne sont qu'une construction. Certains apnéistes confient ne pas identifier de phase de lutte. Le questionnement par l'entraîneur, les pairs ou le pratiquant lui-même se poursuit. Il s'agit d'aider le pratiquant à s'approprier ces zones, à les affiner avec ses propres repères personnels, à leur donner du sens à partir de connaissances théoriques, concernant les effets de l'hypercapnie et de l'hypoxie sur l'organisme par exemple.

Néanmoins, il ne suffit pas de connaître et comprendre pour parvenir à appliquer. C'est le cas de So (35 ans) apnéiste depuis une année et qui évoque les «limites du théorique ». Les connaissances physiologiques acquises cette année à propos de 1 'apnée ${ }^{6}$ lui ont permis d'intégrer ses différentes sensations dans un réseau de significations. Ainsi, la pratiquante a compris, intellectuellement, la zone qu'il lui fallait à présent maîtriser en compétition. Mais en pratique, les mises en œuvre sont encore difficiles. Si l'explication physiologique semble informer l'entrée sensorielle, c'est bien l'expérience qui permet d'affiner des savoir-faire

\footnotetext{
${ }^{6}$ L'apnée au sein de la Fédération Française d'Etudes et de Sports Sous-Marins (FFESSM) se déroule comme la plongée sous-marine. Les pratiquants inscrits dans les clubs affiliés pratiquent et suivent des cours théoriques. Ils sont testés à l'issue de cette formation afin de valider un niveau d'apnée.
} 
perceptifs. So doit encore s'exercer à identifier son état corporel dans cette zone de lutte, affiner ses repères et sa propre échelle esthésiologique, alors même que la lucidité se dérobe du fait de l'hypoxie, afin de sortir de l'eau à temps ${ }^{7}$.

"Faire le récit de son corps» ou construire une «langue du corps» (Andrieu, 2014b) et de ses sensations est un exercice particulièrement complexe pour tout individu. Finalement, par l'apnée, les pratiquants vivent des modelages sensoriels. Ces modelages visent à les rendre plus attentifs à certaines sensations, à les percevoir, à les interpréter ou juger, c'est-à-dire les faire entrer dans un univers de significations partagé. Focalisation de l'attention, « extrémisation » des sensations à l'aide de situations contrastées, partage des ressentis et médiation langagière, utilisation de métaphores pour dire le vécu corporel, apport de connaissances théoriques, proposition puis affinement d'une échelle de ressentis sont autant de démarches destinées à faciliter cette éducation sensorielle et à faire entrer les pratiquants dans un univers partagé. Ainsi, les pratiquants développent une nouvelle compétence perceptive consistant à savoir articuler des épreuves sensorielles avec des raisonnements fondés sur des indices et repères dotés de significations dans un espace de représentation donné (Bessy \& Chateauraynaud, 1995). Enfin, en nommant, en partageant, en classant, se construit peu à peu une culture aquatique et corporelle commune.

\section{Affiner ses savoir-faire perceptifs : une démarche de terrain}

La méthode exposée ici a été imaginée pour résoudre les difficultés rencontrées par Fa (55 ans, compétitrice de niveau international) à ses débuts en apnée. En effet, lors d'apnées dynamiques, $\mathrm{Fa}$, ancienne nageuse, n'éprouve aucune difficulté en ce qui concerne la nage avec une monopalme, les virages, et même la gestion de la performance : "ça se fait tout seul». Les sensations ressenties sont proches de celles connues par la nageuse : cuisses lourdes ou qui brûlent; contractions liées au gainage et à la recherche d'une position hydrodynamique; résistances de l'eau à l'avancement. Elle a déjà appris à interpréter ces sensations au cours de sa carrière de nageuse.

En revanche, à ses débuts en apnée statique, Fa exprime une absence totale de repères, une incapacité à gérer l'évolution de sa performance : "je me couche, je ferme les yeux, et je ne maîtrise rien » $(\mathrm{Fa})$. Elle semble vivre une sorte de déprivation sensorielle, assez typique lorsque l'on débute cette activité. En compétition, elle suit les premiers conseils reçus : « se relâcher ». Mais elle s'abandonne tellement qu'elle part en syncope à 4'30 de performance. Très marquée par cet événement, elle explique ne pas comprendre l'apnée statique, ne rien ressentir, avoir peur que cela se reproduise.

La démarche présentée ici trouve son origine dans cette syncope, et l'impossibilité rencontrée par la pratiquante pour dépasser ses peurs (elle sort systématiquement à 3'30, n'osant aller plus loin). Coach et pratiquante se fixent comme objectif de mieux comprendre ce que vit $\mathrm{Fa}$, pour l'aider à construire des repères sur soi ; des repères internes. Emerge alors l'idée d'une méthodologie nouvelle permettant de documenter puis d'analyser l'expérience corporelle et subjective de l'apnéiste.

\section{a. Une méthode de réflexivité en action}

Un entraîneur est un professionnel de terrain, qui manipule des savoirs aux origines diverses : savoirs scientifiques, savoirs issus de sa formation au monitorat d'apnée, savoirs acquis dans

\footnotetext{
${ }^{7}$ C'est-à-dire avant une perte de lucidité, voire une perte ce contrôle moteur ou une syncope.
} 
son milieu professionnel, savoirs de l'expérience construits lors de ses activités d'encadrant et de pratiquant en apnée. L'enjeu pour ce spécialiste : être efficace face à la complexité des situations de terrain. Face au problème de $\mathrm{Fa}$ à résoudre, il ne s'agit pas d'appliquer une solution toute faite, mais bien de créer une démarche pouvant aider la pratiquante à progresser et dépasser ses blocages induits par la syncope. Ici, Didier, le coach, s'appuie sur : son vécu dans l'activité ; sa connaissance pratique de l'apnée, notamment les quatre phases identifiées précédemment; ses connaissances en Programmation Neuro-Linguistique (PNL) ${ }^{8}$ acquises notamment en formation professionnelle continue.

Quels sont les enjeux de cette méthode ? Il s'agit de proposer une «écoute de soi assistée »: «de favoriser l'hyper-écoute de son corps et de son mental par l'apnéiste, en donnant à l'apnéiste les moyens de verbaliser ses ressentis et son langage interne ${ }^{9}$ directement après plusieurs apnées » (Didier).

Cette méthode repose sur :

- l'identification a priori des durées approximatives des quatre zones d'apnée du pratiquant (confort, travail, effort, lutte). Didier les a construites grâce à sa connaissance des athlètes, notamment leurs performances maximales. Ces indications de temps ne sont pas communiquées au sujet qui, durant toute la séance, est privé de repères chronométriques pour «privilégier sa temporalité interne» (Didier) ainsi que la centration sur son vécu (physiologique, mental, émotionnel).

- l'exploration du cheminement mental, comportemental (mouvements) et sensoriel en apnée au travers de la technique PNL de questionnement des cinq sens dite des «canaux sensoriels », synthétisée sous l'acronyme VAKOG (Visuel, Auditif, Kinesthésique, Olfactif, Gustatif) est adaptée par l'entraîneur. Le recueil du ressenti et du vécu est réalisé sur la base du récit spontané de l'apnéiste soutenu par un questionnement rapide de Didier, essentiellement pour sonder un canal sur lequel le sujet n'aurait rien exprimé en première intention. La grille d'investigation est celle du VAKOG en interrogeant essentiellement trois sens (Visuel, Auditif, Kinesthésique ${ }^{10}$ ) sur les aspects internes et externes, leurs effets positifs ou négatifs, le dialogue interne que cela suscite ou non. Elle relève également un ensemble d'oppositions, graduées sur une échelle, et favorisant la compréhension des états qualitatifs traversés dans le cours de l'apnée :

Aisance / stress ; Maîtrise / lutte ; Facilité / Difficulté ; Relâchement / contraction ; Chaleur / fraîcheur ; Comportements $(\mathrm{K}+/-)$; pensées $(+/$ - ) ; visuel (externe/interne) ; images ; sons (environnant, intérieurs); protocole relaxation ; évocations olfactives - gustatives.

Le nombre d'items est suffisamment large pour que l'entraîneur n'impose pas sa propre grille de lecture et un modèle de perception. Néanmoins, il favorise une exploration bien plus complète que celle réalisée spontanément par le pratiquant. En ce sens, une forme d'éducation sensorielle apparait déjà ici par le fait même de la mise en œuvre de la méthode.

\footnotetext{
${ }^{8}$ Méthode développée dans les années 1970 par John Grinder et Richard Bandler. Démarche pragmatique, la PNL propose des techniques permettant notamment «'exploration du vécu subjectif », c'est-à-dire les états internes et la perception sensorielle du sujet.

${ }^{9}$ Sorte de discours intérieur avec nous-mêmes qui accompagne tout naturellement notre fonctionnement cérébral.

${ }^{10}$ L'olfactif et le gustatif sont peu présents en apnée, si ce n'est éventuellement en évocation.
} 


\begin{tabular}{|l|l|l|}
\hline \multicolumn{1}{|c|}{ Acronyme } & Signification de l'acronyme & Exemples \\
\hline VI & Visuel Interne & $\begin{array}{l}\text { L'apnéiste se forge une image } \\
\text { mentale : des dauphins, une plage } \\
\text { de sable blanc, un paysage enneigé } \\
\text { et la pratique du ski. }\end{array}$ \\
\hline VE & $\begin{array}{l}\text { Regarde les lumières, les reflets, } \\
\text { les carreaux }\end{array}$ \\
\hline AI & Auditif Interne & $\begin{array}{l}\text { Air de musique en tête, choisi } \\
\text { pour relaxer, donner de l'énergie. }\end{array}$ \\
\hline AE & Auditif Externe & $\begin{array}{l}\text { Bruits de l'eau, clapotis, } \\
\text { conversations alentour }\end{array}$ \\
\hline KI & Kiné Interne & $\begin{array}{l}\text { Sentir le relâchement musculaire, } \\
\text { tension dans la nuque, mâchoire } \\
\text { crispée, le diaphragme qui spasme }\end{array}$ \\
\hline KE (toucher) & Kiné Externe & $\begin{array}{l}\text { La température de l'eau sur la } \\
\text { peau, le contact de l'entraîneur sur } \\
\text { le dos } \\
\text { «tu es capable », «tout va bien, } \\
\text { relâche toi », «je suis mal parti », } \\
\text { «qu'est-ce que je fais ici ? » }\end{array}$ \\
\hline DI & Dialogue Interne &
\end{tabular}

Tableau 1 : Présentation des acronymes avec des exemples de données recueillies suite à des apnées statiques

\section{b. La mise en cuvre du protocole}

Dans le bassin, l'apnéiste déroule son protocole usuel de préparation (mentale et technique) et débute sont apnée. Il est demandé à l'athlète de se projeter mentalement et techniquement dans un objectif de performance maximale comme en compétition. Le postulat étant que cette consigne induira de sa part un comportement (dedans et dehors) identique ou proche de celui qui l'anime communément dans ce contexte.

L'apnéiste est interrompu en fin de la première zone d'aisance pour un recueil très bref (trente secondes à une minute maximum) «à chaud », sans espace pour la prise de recul réflexive ou la mise en forme de son récit, d'une part pour en garantir l'immédiateté et l'authenticité, d'autre part pour que l'athlète reste mentalement en «mode perf apnée » (Didier). Puis il repart en concentration et préparation pour s'engager, une fois prêt, dans une nouvelle apnée longue.

A l'initiative du coach, il est ensuite interrompu successivement en fin de zone de travail, puis de zone d'effort, et interrogé une dernière fois lorsqu'il met lui-même fin à son apnée.

L'apnéiste est donc interrogé quatre fois. Au final, le pratiquant aura donc réalisé quatre apnées, en étant interrompu au cours des trois premières apnées à des moments différents (phase d'aisance, de travail puis d'effort). La dernière apnée est menée le plus loin possible. Enfin, ce protocole est reproduit trois fois à quelques semaines d'intervalle.

Le coach remplit au bord du bassin sa grille d'investigation. Les données recueillies sont des données en première personne, bien que produites par un outil construit en troisième personne. 
Ces données vont par ailleurs être traitées puis interprétées dans un premier temps par l'entraîneur, qui va analyser les comportements, compétences et habiletés mises en exergue pour identifier un mode opératoire propre à l'athlète. Il s'agit donc d'une interprétation en troisième personne permettant d'identifier la stratégie mentale de l'athlète ainsi que son fonctionnement sensoriel préférentiel. Ensuite, ces analyses seront proposées et discutées avec le pratiquant pour validation ou invalidation, affinements éventuels. Enfin, une stratégie pourra être co-construite afin d'améliorer les performances en apnée statique (stratégie mentale, construction d'un ancrage, construction de repères sensoriels précis, etc.).

c. Analyse des données recueillies avec Fa

Trois séances d'investigation ont été réalisées avec l'athlète, à quelques semaines d'intervalle à chaque fois. Nous les nommerons Observation 1 (11/2009), Observation 2 (12/2009) et Observation $3(01 / 2010)$. 


\section{Observation 1}

\section{Phase 1 Temps : 2’30}

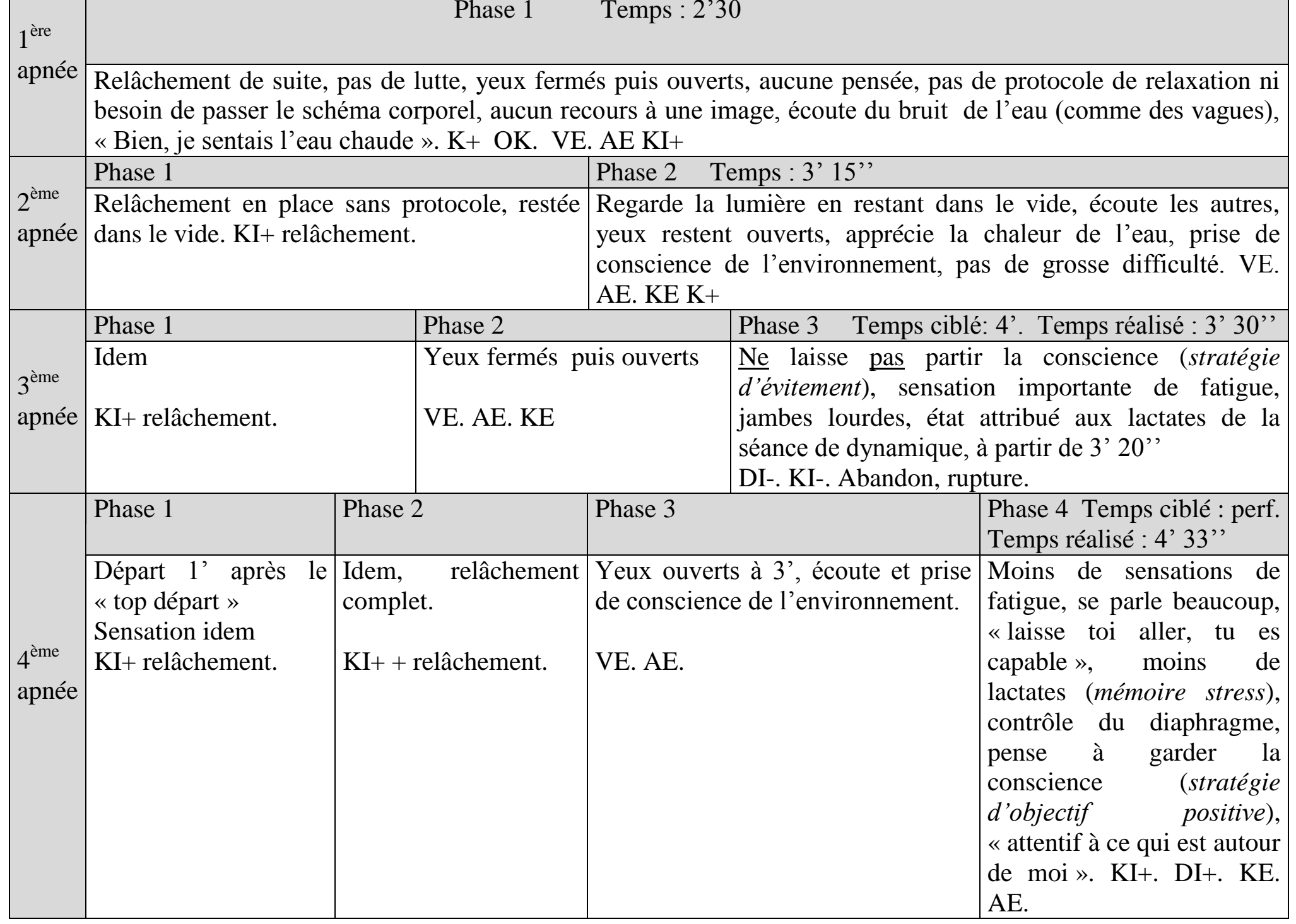

Tableau 2 : Exemple d'une fiche de recueil

Analyse de la phase $1:$ l'aisance

Lors des différentes apnées statiques réalisées avec ce protocole (Observations 1, 2 et 3), des points communs émergent chez l'athlète au cours de cette première phase d'apnée : le relâchement complet, le plaisir, la facilité, la légèreté.

Ce relâchement est parfois obtenu par un protocole de relaxation, une rotation de conscience ( « passe en revue le schéma corporel »), une intention explicite : « rester à l'écoute du corps », «Pense à la décontraction du corps ». D'autres fois, le protocole n'est pas nécessaire : «Relâchement de suite, pas de lutte, yeux fermés puis ouverts, aucune pensée, pas de protocole de relaxation ni besoin de passer le schéma corporel, aucun recours à une image, écoute du bruit de l'eau (comme des vagues) », «Bien, je sentais l'eau chaude » 
Analyse de la phase 2 : le travail, la production d'image mentale

Cette phase débute autour de 2' d'apnée chez Fa. «Ça tire un peu ». Fa essaie parfois, au cours de cette phase, de se forger une image mentale positive. Elle ouvre les yeux, se souvient de «la lumière, les reflets, la couleur bleue». Dans cette phase, parfois, elle «se forge une image de nage en monopalme, ondulation, image du dauphin ». D'autres fois, elle demeure relâchée mais en se connectant à l'environnement extérieur : "Regarde la lumière en restant dans le vide, écoute les autres, yeux restent ouverts, apprécie la chaleur de l'eau, prise de conscience de l'environnement, pas de grosse difficulté » (Observation 3).

Analyse de la phase 3 : l'effort, un moment de stress récurrent

Dans cette phase, aux alentours de 3'30, un moment de stress est récurrent. Fa a peur et veut éviter de revivre la mauvaise expérience des Championnats de France. Elle se parle : «ne laisse pas partir la conscience ».

Au fur et à mesure des séances d'entraînement et d'échanges avec son entraîneur, la teneur de cette phase évolue. Si le moment de stress demeure, Fa parvient à en prendre conscience, pour s'en détacher et « repartir dans la concentration». Elle se focalise sur les battements de son cœur : «être à l'écoute du corps, concentré sur soi-même », mais le stress revient, une sorte de « renfermement sur soi » contre-productif (Observation 2)

Quelques semaines plus tard, le vécu de cette phase est encore différent. Fa se centre sur la prise de conscience de l'environnement, «attentive à ce qui est autour de moi » (Observation 3) : "Analyse le moment de toucher le mur. Yeux ouverts, rester relâchée, écoute l'environnement extérieur, ressentir la chaleur, eau qui porte, bercée par la vague. Regarde les reflets, zone globale d'aisance tout le long ».

Analyse de la phase 4 : lutte, concentration et reconnexion?

Les données sont moins nombreuses pour illustrer cette dernière phase. Sur douze apnées finalement verbalisées, deux sont suffisamment longues pour que $\mathrm{Fa}$ atteigne la phase 4 (audelà de 4 minutes pour elle). Fa se parle beaucoup « laisse toi aller, tu es capable ». Il s'agit de rester lucide «toujours attentive à ce qui est autour de moi », tout en contrôlant certaines sensations négatives (le diaphragme).

\section{d. Analyse prospective du coach}

Didier transcrit ensuite tous les éléments de façon neutre. Il veille à ne porter aucun jugement de valeur sur l'expression, les comportements, les ressentis, les émotions, livrés par l'athlète. Puis il analyse, émet des hypothèses et des propositions, qu'il partagera avec l'apnéiste. Ce dernier se retrouve en position dissociée de l'expérience vécue, tel un observateur extérieur. Cette phase de décodage du vécu de l'expérience débouche sur l'identification de points forts à valoriser, de points sensibles à interroger et d'hypothèses prospectives de travail formulées par le coach. Cette étape aboutit à la production d'un récit en «troisième personne ». 


\begin{tabular}{|c|c|}
\hline Points validés & $\begin{array}{l}\text { «Concentration sur les battements du cœur »: Se référer au rythme } \\
\text { cardiaque bonne piste car cela renvoie à la notion de rythme de } \\
\text { nage, façon de dynamiser l'apnée statique en la rythmant, lui donner } \\
\text { des aspérités. } \\
\text { Fa parvient à stopper un dialogue interne négatif ( « ne pas laisser } \\
\text { partir la conscience } »=\text { évitement et peur) et des sensations } \\
\text { négatives (fatigue, jambes lourdes), d'une apnée à l'autre. Instaure un } \\
\text { dialogue interne positif : «laisse toi aller, tu es capable », "pense à } \\
\text { garder la conscience »= stratégie d'objectif positif. }\end{array}$ \\
\hline Points sensibles & $\begin{array}{l}\text { Accepter l'image du championnat de France (et de la syncope) sans } \\
\text { se focaliser et en sachant qu'elle s'estompera avec le temps. La } \\
\text { mettre dans le décor et de plus en plus en arrière-plan jusqu'à ce que } \\
\text { par la distanciation elle ne soit plus visible. } \\
\text { Croyances limitantes en début de performance (n'a pas fait son } \\
\text { protocole habituel de préparation à la performance) }\end{array}$ \\
\hline $\begin{array}{l}\text { Pistes de travail et } \\
\text { d'amélioration }\end{array}$ & $\begin{array}{l}\text { Dans les moments de «stress, de doute » ou de mobilisation de } \\
\text { l'esprit par une image ou des pensés parasites, recourir à l'image de } \\
\text { référence personnelle pour se remettre en phase avec } \\
\text { l'environnement et se centrer sur «l'ici et maintenant» pour rester } \\
\text { pleinement dans sa performance du jour. } \\
\text { Mobiliser une image de détente (VI), la placer dans le décor } \\
\text { extérieur, puis vérifier consciemment les sensations corporelles (KI) } \\
\text { Rester dans «l'ici et maintenant» de la performance, cesser } \\
\text { d'idéaliser des sensations ressenties lors de performances passées, et } \\
\text { chercher à les revivre (eau chaude, détente) / ou cesser de craindre } \\
\text { des sensations passées désagréables et chercher à les éviter. Etre } \\
\text { orienté vers l'avenir. } \\
\text { Utiliser en compétition les repères externes lorsqu'il faut se } \\
\text { reconnecter : le «top sécu }{ }^{11} \text {. }\end{array}$ \\
\hline
\end{tabular}

Tableau 3 : Exemple de tableau d'analyse construit par l'entraîneur et soumis au pratiquant

\section{e. Homologation conjointe coach/athlète}

${ }^{11}$ Si l'athlète en compétition annonce qu'il espère réaliser 4 minutes, alors l'apnéiste de sécurité, une minute avant l'objectif annoncé, réalise un "top sécu" (toucher ou pincer l'épaule par exemple). L'apnéiste doit répondre à ce signal kinesthésique externe par le signe $\mathrm{OK}$. Jusqu'à l'objectif annoncé, l'apnéiste de sécurité réalisera un "top sécu" toutes les 30 secondes, puis passées 4', toutes les 15 secondes. Si l'athlète ne répond pas par le signe $\mathrm{OK}$, il est sorti de l'eau et sa performance n'est pas validée. Ce procédé doit permettre de tester la lucidité de l'athlète. 
Dans un troisième temps, le coach soumet au jugement de l'athlète ses hypothèses, conclusions et pistes d'amélioration. L'enjeu consiste à vérifier si l'interprétation du coach est juste quant à l'expérience vécue par Fa. Il s'agit ensuite de co-construire une stratégie d'apprentissage en validant, précisant ou ajustant les pistes de travail évoquées précédemment. Dans ce cas, le lien et la confiance coach/athlète sont tels que Fa fut réceptive aux éclairages et conseils qu'elle mit en œuvre rapidement.

Mais il y a aussi des interprétations non communiquées à la championne, expression du point de vue en troisième personne de l'expert mais aussi de la perception en première personne de l'entraineur qui tient son journal sur ses hypothèses et ses croyances afin de valider des techniques à retravailler à la prochaine séance.

Après ces trois séances de travail, mais surtout des entrainements au cours desquels Fa s'est peu à peu approprié les points co-construits avec le coach, le blocage à 3'30 a été dépassé. Fa a finalement défini un "mode opératoire » mental et technique qu'elle applique encore aujourd'hui en apnée statique. Elle a d'ailleurs remporté en 2011 aux Championnats du Monde une médaille d'argent en apnée statique avec la performance de 5'37, son record personnel en compétition. Les progrès sont visibles avec cette performance chiffrée, mais l'aspect qualitatif de ces progrès est moins visible mais peut être encore plus important. En effet, Fa a ainsi gagné en maîtrise et en confiance. L'épreuve de statique impactant moins les autres épreuves du combiné ${ }^{12}$ par de la fatigue excessive ou du stress.

\section{Conclusion}

En apnée, les pratiquants vivent des modelages sensoriels, explicites ou implicites, destinés à les rendre plus attentifs à certaines sensations, à les interpréter ou juger, c'est-à-dire les faire entrer dans un univers de significations partagé. Ils construisent des savoir-faire perceptifs entendus comme la capacité à utiliser sa sensorialité (les ressentis corporels ici) pour agir et prendre des décisions (Schirrer \& Paintendre, 2017).

Les entraînements hebdomadaires d'apnéistes ordinaires donnent à voir des processus d'éducation de l'attention et de la sensibilité, afin d'aider chacun à écouter son propre corps, au service de la régulation de sa performance, ou parfois simplement pour son bien-être. Alors que les gestes techniques s'automatisent, l'apnéiste améliore l'acuité de la perception des sens pour identifier les «bonnes » et «mauvaises » sensations (notamment à l'approche du seuil hypoxique). Il devient capable d'accueillir des sensations a priori désagréables sans stresser, parce qu'il a appris à les reconnaître, à les intégrer dans son expérience, à leur donner du sens. Il a appris à s'orienter dans ce monde de sensations, qui deviennent aides à la décision, moyen de jugement et de régulation de ses propres actions.

Une démarche originale proposée à une apnéiste confirmée, rencontrant des difficultés de repérage en apnée statique, donne à voir l'expérience vécue en apnée statique. Cette expérience subjective se révèle être un ensemble complexe de ressentis corporels, de langage interne et de croyances ${ }^{13}$ auquel l'apnéiste apprend à se rendre attentif, afin de co-construire,

${ }^{12}$ En compétition, les athlètes sont classés par discipline : dynamique avec palmes, sans palmes et statique. Ils sont aussi classés au combiné des trois disciplines : on additionne alors les points obtenus dans chaque discipline.

${ }^{13}$ "Il s'agit de l'attention (à quoi et comment je suis attentif), du langage interne (ce que je me dis), des croyances (ce que je pense juste et possible de faire), des valeurs (ce que je crois bien de faire). »(Gaillard, 2011, pp. 81-82). 
avec le coach, une stratégie efficace en statique. L'entraineur, par cette verbalisation outillée avec le VAKOG, oscille entre la volonté de recueillir des données sur l'expérience à des instants précis (au moment de l'interruption), et la volonté de développer une collaboration entre le pratiquant et lui-même dans l'analyse de l'activité (dans l'analyse après coup). De fait, cette exploration outillée amène le pratiquant à explorer certains canaux sensoriels jusque-là peu pris en compte. Il participe ainsi d'une reconfiguration sensorielle en amenant le pratiquant à se centrer sur certaines sensations, qui améliorent la performance ou aident à mieux se situer, afin de ne plus partir en syncope. Finalement, une stratégie d'apprentissage est co-construite par le pratiquant et son entraîneur, afin d'affiner des savoir-faire perceptifs et construire des stratégies mentales destinées à améliorer les performances en apnée statique. Plus qu'un savoir-faire perceptif, c'est une compétence perceptive qui est développée ici, entendue comme capacité à mobiliser à bon escient ses ressources, à juger pour agir face à une diversité de situations.

\section{Références}

Andrieu, B., \& Burel, N. (2014). La communication directe du corps vivant. Une émersiologie en première personne. Hermès, 68(1), 46-52.

Andrieu, B. (2013a). Le corps en première personne : une écologie pré-motrice. Movement \& Sport Sciences, 81(3), 1-3.

Andrieu, B. (2013b). Sentir son corps en première personne : une écologie pré-motrice. Movement \& Sport Sciences, 81(3), 91-99.

Andrieu, B. (2014a). Une cosmotique immersive. Pour une écologie corporelle en première personne. Nature \& Récréation, 1, 20-24.

Andrieu, B. (2014b). Le corps-chronique en première personne : s'autosanter par les récits de sa maladie. Thérapie psychomotrice et recherches, 77(47), 4-15.

Andrieu, B. (2016). Sentir son corps vivant. Emersiologie 1. Paris : Vrin.

Becker, H. (2012, $1^{\text {ère }}$ éd. 1963). Outsiders. Etudes de sociologie de la déviance. Paris : Métailié.

Berthoz, A., \& Andrieu, B (Eds). (2011). Le corps en acte. Centenaire M. Merleau-Ponty. Nancy : PUN.

Bessy, C., \& Chateauraynaud, F. (1995). Experts et faussaires. Pour une sociologie de la perception. Paris : Editions Pétra.

Bessy O. (2012). The North Face. Ultra-Trail du Montblanc. Un mythe, un territoire, des hommes, éd. Petit montagnard-Autour du Mont-Blanc.

Candau, J. (2005). Vin, arômes, couleurs et descripteurs sensoriels. Quel partage de la dégustation ? Médiation \& Information, 23, 23-38.

Chavaroche, L. (2018). The emersion of sensation in slacklining. In Andrieu, B., Parry, J., Porrovecchio, A., Sirost, O. Body ecology and emersive leisure (pp. 216-226). Routledge : Abingdon.

Corneloup, J. (2011). La forme transmoderne des pratiques récréatives de nature. Développement durable \& Territoires [en ligne], 3(2), consulté le 02 septembre 2017.

Fricker, O. (2015). Historique de l'apnée fédérale. In M. Schirrer (Ed.), S'immerger en apnée. Cultures motrices et symbolismes aquatiques (pp. 61-83). Paris: L'Harmattan, coll. « Mouvements des savoirs ».

Frolla, P., \& Raveneau, G. (2006). Au plus profond de soi. Ethnologie française, 36(4), 681683.

Gaillard, J. (2011). Apprentissage technique et prise en compte de la sensibilité. In B. Huet \& N. Gal-Petitfaux (Eds.). L'expérience corporelle (pp. 77-96). Paris : éd. Revue EP\&S, coll. « Pour l'action ». 
Gélard, M.-L. (2013). Corps sensibles. Usages et langages des sens. Nancy : PUN.

Kalaora, B. (2001). A la conquête de la pleine nature. Ethnologie Française, 31(4), 591-597.

Le Breton, D. (1992). La sociologie du corps. Paris : PUF.

Lemaître, F. (Ed.). 2011. L'apnée : de la théorie à la pratique. Rouen : PURH.

Pasche, K., Bertrand, B. (2013). Arts de vie sauvage, gestes premiers. Escalquens : Terran Editions.

Petitmengin, C. (2004). Peut-on anticiper une crise d'épilepsie ? Explicitation et recherche médicale. Expliciter, 57, 1-24.

Récopé, M. (2001). L'adaptation au cœur des apprentissages. In M. Récopé (Ed.). L'apprentissage (pp. 11-30). Paris : éd. Revue EP\&S, coll. « Pour l'action ».

Sayeux, A.-S. (2008). Surfeurs, l'être au monde. Une analyse socio-anthropologique. Rennes : Presses Universitaires de Rennes.

Schirrer, M. \& Paintendre, A. 2017. Rapport au corps et savoir-faire perceptif dans les activités physiques et sportives. In G. Gogérino, G. (Ed.). Rapport au corps, genre et réussite en EPS (pp. 275-290). Clapiers : AFRAPS.

Schirrer, M. (Ed.). (2015a). S'immerger en apnée: cultures motrices et symbolismes aquatiques. Paris : L'Harmattan.

Schirrer, M. (2015b). Mesure et démesure en apnée sportive. L'expérience de la syncope. Actes du congrès international Démesure de l'Association française d'ethnologie et d'anthropologie (AFEA). [En ligne] https://demesure.sciencesconf.org/

Schirrer, M. (2015c). Apnée. In B. Andrieu (Ed.). Vocabulaire International de philosophie du sport, Tome 2 : les nouvelles recherches (pp. 213-232). Paris: L'Harmattan, coll. " Mouvements des savoirs ».

Schirrer, M. (2018). The emersion of blackout in freediving : moderation and immoderation. In Andrieu, B., Parry, J., Porrovecchio, A., Sirost, O. Body ecology and emersive leisure (pp. 203-215). Routledge : Abingdon.

Vermersch, P. (1993). L'entretien d'explicitation. Paris : ESF.

Vigarello, G. (2014). Le sentiment de soi : Histoire de la perception du corps (XVIe-XXe siècle). Paris : Seuil.

Wacquant, L. (1989). Corps et âme. Actes de la Recherche en Sciences Sociales, 80, 33-67.

Wathelet, O. (2013). Ethnographie cognitive des sens. In Gélard, M.-L. (Ed.). Corps sensibles. Usages et langages des sens (pp. 303-317). Nancy : PUN. 